\title{
The Solution of a Class of Integral Equations
}

\author{
G. E. LATTA
}

Communicated by D. GILBARG

1. Introduction. One of the standard methods for dealing with boundary value problems for partial differential equations is to reformulate the problem as an integral equation. There are many advantages to this approach, even if it turns out to be too difficult to solve the integral equations in closed form.

To mention one or two, we frequently do not need the complete solution of the boundary value problem, but only a derivative at the boundary, or an integral of certain boundary data, and the solution of the integral equation may give this directly. A second advantage lies in the fact that the integral equation furnishes a problem in fewer dimensions than the original problem.

The purpose of this paper is to develop a method for obtaining the solution of a certain class of integral equations by showing that the required solution satisfies an ordinary differential equation, and setting up the appropriate boundary value problem for the solution of the differential equation. The method used will be made clear by means of examples, several of which have been solved by such methods as the Wiener-Hopf procedure, and function theoretic considerations.

As an illustration of the reduction to an integral equation, consider the following boundary value problem. Find $\varphi(x, y)$ so that

$$
\Delta \varphi-\varphi=0 \quad \Delta=\frac{\partial^{2}}{\partial x^{2}}+\frac{\partial^{2}}{\partial y^{2}}
$$

in the $x, y$ plane outside the slit $y=0, a \leqq x \leqq b$, so that

$$
\begin{aligned}
\varphi(x, 0) & =g(x) \quad a<x<b \\
\varphi(x,-y) & =\varphi(x, y) .
\end{aligned}
$$

Now, the fundamental solution of (1) in the complete $x, y$ plane is $K_{0}(r)$, the Bessel function of the second kind with imaginary argument, where 
$r^{2}=\left(x-x_{0}\right)^{2}+\left(y-y_{0}\right)^{2}$. It follows from the properties of the fundamental solution that the solution of

$$
\Delta \varphi-\varphi=-2 \pi f(x, y)
$$

in the whole $x, y$ plane, subject to the condition that $\varphi(x, y) \rightarrow 0$ as $|x|,|y| \rightarrow \infty$, is given by

$$
\varphi(x, y)=\iint_{x, y} f(Q) K_{0}\left(r_{P Q}\right) d Q
$$

with

$$
P=(x, y), \quad Q=(\xi, \eta), \quad d Q=d \xi d \eta .
$$

If we now replace our problem (1) by the problem (2), and require $f(x, y)$ to vanish outside the slit $a<x<b, y=0$, and ask for the solution in the whole plane, we obtain formally

$$
\varphi(x, y)=\int_{a}^{b} f(\xi) K_{0}\left(\sqrt{\left.(x-\xi)^{2}+y^{2}\right)} d \xi,\right.
$$

which solves the original problem provided $f(x)$ is chosen so that

$$
g(x)=\int_{a}^{b} f(\xi) K_{0}(|x-\xi|) d \xi \quad a<x<b .
$$

Thus the original mixed boundary value problem has been reduced to the equivalent integral equation of the first kind (4).

From (3) we see that $f(x)$ is proportional to $\frac{\partial \varphi}{\partial y}(x, 0)$; also, the solutions of (1) satisfy the maximum principle. Thus if $g(x)>0$, for example, and $\varphi \rightarrow 0$ as $x, y \rightarrow \infty$, then $\max \phi(x, y)$ occurs on $y=0, a<x<b$, so that $\frac{\partial \varphi}{\partial y}(x, 0) \leqq 0$, or, of constant sign in $(a, b)$. This will be of value in later applications.

We now turn to the problem of solving such integral equations. The next section illustrates on a well known example ${ }^{1)}$ the method used in this paper.

2. The Integral Equation $\int_{-1}^{1} \ln |x-t| f(t) d t=g(x), \quad-1<x<1$. The solution of the boundary value problem

$$
\Delta \varphi(x, y)=0
$$

in the $x, y$ plane, subject to

$$
\begin{aligned}
\varphi(x, 0) & =g(x), \quad-1<x<1 \\
\varphi(x,-y) & =\varphi(x, y),
\end{aligned}
$$

1)The examples of Section 2 are readily solved by the use of conformal mapping, but these methods are not available for other differential equations. 
can be represented as

$$
\varphi(x, y)=\int_{-1}^{1} \ln \left(\sqrt{(x-t)^{2}+y^{2}}\right) f(t) d t
$$

where $f(x)$ is to be found from the integral equation of the first kind,

$$
\int_{-1}^{1} \ln (|x-t|) f(t) d t=g(x), \quad-1<x<1 .
$$

Here $f(x)=\frac{1}{\pi} \frac{\partial \varphi}{\partial y}(x, 0)$ in $-1<x<1$, and since $\varphi(x, y)$ satisfies the maximum principle, we again infer that $f(x)$ is of one sign if $g(x)$ is also of one sign.

The first step is to note that the kernel of $(5), K(x)=\ln |x|$, satisfies an ordinary differential equation

$$
x K^{\prime}(x)-1=0 .
$$

This enables us to express $\Gamma x f$ in terms of $\Gamma f$, where we write

$$
\Gamma f(x)=\int_{-1}^{1} \ln |x-t| f(t) d t
$$

Thus

$$
\begin{gathered}
\Gamma x f=\int_{-1}^{1} t \ln |x-t| f(t) d t \\
=-\int_{-1}^{1}(x-t) \ln |x-t| f(t) d t+x \Gamma f \\
\frac{d}{d x} \Gamma x f=\Gamma^{\prime} x f=x \Gamma^{\prime} f-\int_{-1}^{1} f(t) d t \\
\Gamma^{\prime} x f=x \Gamma^{\prime} f-\int_{-1}^{1} f(t) d t .
\end{gathered}
$$

This is the first of two identities which play a fundamental role in the solution of the integral equation. The second identity utilizes the fact that the kernel is a function of the difference $x-t$. Thus, if $y(x)$ is any differentiable function which vanishes at $x= \pm 1$, and $K(u)$ is any integrable function, then

$$
\int_{-1}^{1} K(x-t) y(t) d t=\int_{-1}^{x} K(x-t) y d t+\int_{x}^{1} K(x-t) y d t
$$

Let

$$
\int_{0}^{u} K(v) d v=h(u), \quad \text { so that } \quad h(0)=0
$$


Integration by parts gives

$$
\begin{aligned}
\int_{-1}^{1} K(x-t) y d t=-\left.h(x-t) y\right|_{-1} ^{x}- & \left.h(x-t) y\right|_{x} ^{1} \\
& +\int_{-1}^{x} h(x-t) y^{\prime} d t+\int_{x}^{1} h(x-t) y^{\prime} d t
\end{aligned}
$$

and the integrated terms vanish. Differentiation with respect to $x$ gives

$$
\frac{d}{d x} \int_{-1}^{1} K(x-t) y d t=\int_{-1}^{1} K(x-t) y^{\prime} d t
$$

and in terms of our integral operator $\Gamma$,

$$
\Gamma^{\prime} y=\Gamma y^{\prime}
$$

for arbitrary differentiable $y(x)$ such that $y(-1)=y(1)=0$. This is the second identity used in the reduction of the integral equation to an ordinary differential equation. We observe that equation (6) expresses precisely the fact that the kernel of the integral equation is $\log |x|$, while (8) remains true for any difference kernel.

Now we note that the integral equation $\Gamma f=g(x)$ has a unique solution, since $\Gamma f=0$ implies $f(x)=0$ from the maximum principle. In particular, then, if $g(x)$ is even in $x$, so is $f(x)$. For reasons which will be made clear later, we start with the special equation

$$
\Gamma f=1 \quad \text { in } \quad-1<x<1 .
$$

Then (6) gives

$$
\Gamma^{\prime} x f=-\int_{-1}^{1} f(t) d t=-\mu_{0}
$$

and thus

$$
\Gamma x f=-\mu_{0} x+C
$$

We may put $C=0$ since here $f(x)$ is even, so that $\Gamma x f$ is odd. The computation of $\Gamma x^{2} f$, and of higher powers $\Gamma x^{n} f$, proceeds in the same way, but we need only $\Gamma x^{2} f$, namely

$$
\Gamma x^{2} f=-\mu_{0} \frac{x^{2}}{2}+\lambda
$$

So far, we have merely required that $f(x)$ be integrable over $(-1,1)$ in order that the expression $\Gamma f$ be defined. On the other hand, $f(x)$ has derivatives of all orders in $-1<x<1$, and behaves like $(1-|x|)^{-\frac{1}{2}}$ as $|x| \rightarrow 1$, as can readily be shown from general function theoretic considerations. We take a different 
point of view, and look for a solution of $\Gamma f=1$ in the class of functions $\{f(x)\}$ for which $f(x)$ is differentiable in $-1<x<1$, and $\left(1-x^{2}\right) f(x) \rightarrow 0$ as $|x| \rightarrow 1$. This permits us to take

$$
y(x)=\left(1-x^{2}\right) f(x)
$$

as a suitable function to use in (8). We get

$$
\Gamma^{\prime} y=\Gamma y^{\prime}=\frac{d}{d x}\left\{\Gamma f-\Gamma x^{2} f\right\}=\mu_{0} x=-\Gamma x_{J}
$$

or,

$$
\Gamma\left(y^{\prime}+x f\right)=0 .
$$

This in turn implies

$$
y^{\prime}=-x f .
$$

Elimination of $y(x)$ between (9) and (10) yields

$$
\left(1-x^{2}\right) f^{\prime}-x f=0
$$

and

$$
f=\frac{C}{\sqrt{1-x^{2}}} .
$$

That this is the correct solution can easily be checked. It is clear that this procedure will work if we can guarantee that $f(x)$ has at most an algebraic singularity at $x= \pm 1$. Otherwise we cannot conclude that $\left(1-x^{2}\right) f(x) \rightarrow 0$ as $|x| \rightarrow 1$. On the other hand, we can eliminate the assumption that $\left(1-x^{2}\right)$ $f(x) \rightarrow 0$ as $|x| \rightarrow 1$. If we merely assume $f(x)$ integrable and even, we may take

$$
y(x)=\int_{-1}^{x} t f d t .
$$

Then

$$
\Gamma^{\prime} y=\Gamma y^{\prime}=\Gamma x f=-\mu_{0} x,
$$

and integrating,

$$
\Gamma y=-\frac{\mu_{0} x^{2}}{2}+A=\Gamma x^{2} f+(A-\lambda) \Gamma f .
$$

Hence

$$
\begin{aligned}
y & =x^{2} f+(A-\lambda) f, \\
y^{\prime} & =x f
\end{aligned}
$$


Eliminating $y(x)$, we obtain

$$
\left(x^{2}+A-\lambda\right) f^{\prime}+x f=0
$$

so that $f(x)$ has at worst an algebraic singularity for finite $x$. Alternately, from

$$
y=\left(x^{2}+A-\lambda\right) f \quad \text { and } \quad y(1)=y(-1)=0
$$

we have either $f( \pm 1)=0$ or $A-\lambda=-1$ and $\left(x^{2}-1\right) f(x) \rightarrow 0$ as $|x| \rightarrow 1$. Since it simplifies the analysis at times, we find it more convenient to include this property of the function $f(x)$ as an assumption, there being no loss in generality.

It now becomes clear why we started with the special problem $\Gamma f=1$. When we use equation (6) to compute $\Gamma x f$ in terms of $\Gamma f$,

$$
\Gamma^{\prime} x f=x \Gamma^{\prime} f-\int_{-1}^{1} f d t
$$

we have an integral equation to start with, say $\Gamma f^{*}=g(x)$ in $-1<x<1$.

Then (6) may be regarded as an ordinary differential equation for $\Gamma x f^{*}$,

$$
\frac{d}{d x} \Gamma x f^{*}=x g^{\prime}(x)-\mu_{0}, \quad \mu_{0}=\int_{-1}^{1} f^{*} d t
$$

Integration gives $\Gamma x f^{*}$ in terms of the special functions $x, 1$, and $g(x)$ again. In order to perform the calculations following (9), we need a representation of 1 as $\Gamma f$ for some $f(x)$. Accordingly, we must first obtain this representation, or, in other words, solve the equation $\Gamma f=1$, before proceeding to other right hand sides. The solutions of all other equations $\Gamma f^{*}=g(x)$ depend on the special function $f_{0}(x)=1 / \sqrt{1-x^{2}}$ obtained from the solution of $\Gamma f=1$. As an illustration, consider

$$
\Gamma g=\cosh x
$$

Then $g(x)$ is even in $x$, and

$$
\begin{gathered}
\Gamma x g=x \cosh x-\sinh x-\mu_{0} x, \quad \mu_{0}=\int_{-1}^{1} g d t \\
\Gamma x^{2} g=x^{2} \cosh x-2 x \sinh x+2 \cosh x-\frac{\mu_{0}}{2} x^{2}+A .
\end{gathered}
$$

We are going to need a representation for $x \cosh x$ as $\Gamma k$ for some $k(x)$, and as seen from (11), this is equivalent to knowing $h(x)$ for which $\Gamma h=\sinh x$. We therefore introduce an auxiliary integral equation

$$
\Gamma h=\sinh x,
$$


and then $h(x)$ is odd in $x$, and

$$
\begin{gathered}
\Gamma x h=x \sinh x-\cosh x+B, \\
\Gamma x^{2} h=x^{2} \sinh x-2 x \cosh x+2 \sinh x-\nu_{1} x, \\
\nu_{1}=\int_{-1}^{1} x k d x .
\end{gathered}
$$

Choosing

$$
y_{1}=\int_{-1}^{x} t g d t, \quad y_{2}=\int_{-1}^{x} h d t
$$

we find

$$
\begin{aligned}
& y_{1}=x h-g-(B-D) f_{0}-\frac{\mu_{0}}{2} f_{2}, \\
& y_{1}^{\prime}=x g,
\end{aligned}
$$

where for convenience we have set $\Gamma f_{n}=x^{n}$.

$$
\begin{aligned}
& y_{2}=g+E f_{0}, \\
& y_{2}^{\prime}=h .
\end{aligned}
$$

Elimination of $y_{1}, y_{2}$ gives a pair of first order ordinary non-homogeneous differential equations for the unknown functions $g, h$. The non-homogeneous terms involve the special function $f(x)$, already determined. The functions $g, h$ can be obtained by other methods, perhaps more straightforwardly, but our formulation gives a systematic method for solving certain integral equations for which no other methods have proven practical. For completeness, we include the necessary computation for the evaluation of the unknown constants appearing in (12) and (13).

Since $\Gamma g=\cosh x>0$ in $(-1,1)$, we have $g(x) \leqq 0$ in $(-1,1)$ from the maximum principle. This permits us to define

$$
\begin{aligned}
& y_{3}^{\prime}=g+\lambda f_{0} \\
& y_{3}=\int_{0}^{x}\left(g+\lambda f_{0}\right) d t,
\end{aligned}
$$

choosing $\lambda$ so that $y_{3}(1)=0$. Then $y_{3}(-1)=0$ also since $y_{3}(x)$ is odd. Using $\Gamma^{\prime} y_{3}=\Gamma y_{3}^{\prime}$, we obtain

$$
\begin{aligned}
& y_{3}=h+\lambda f_{1} \\
& y_{3}^{\prime}=g+\lambda f_{0}
\end{aligned}
$$

and thus, eliminating $y_{3}$,

$$
g=h^{\prime}+\lambda\left(f_{1}^{\prime}-f_{0}\right) .
$$


From (12) and (13) we have

$$
\begin{gathered}
x h^{\prime}+h-g^{\prime}-x g=(B-D) f_{0}+\frac{\mu_{0}}{2} f_{2}^{\prime}, \\
g^{\prime}+E f_{0}=h .
\end{gathered}
$$

Consistency among (14), (15), (16) gives

$$
\lambda=\mu_{0} \ln 2 .
$$

Now $y_{2}=g+E f_{0}, y_{3}=h+\lambda f_{1}$, and each $y_{i}$ vanishes at $x= \pm 1$. Rewriting (14) and (16) in terms of $y_{2}, y_{3}$ gives

$$
\begin{aligned}
& y_{2}^{\prime}=y_{3}-\lambda f_{1}, \\
& y_{3}^{\prime}=y_{2}+(\lambda-E) f_{0} .
\end{aligned}
$$

Taking Laplace transforms, we get

$$
\begin{aligned}
& \mathscr{L}\left\{f_{0}\right\}=\int_{-1}^{1} e^{-s x} f_{0}(x) d x=-\frac{1}{\ln 2} I_{0}(s), \\
& \mathscr{L}\left\{f_{1}\right\}=-s I_{1}(s),
\end{aligned}
$$

where $I_{0}(s), I_{1}(s)$ are Bessel functions of the first kind with imaginary argument.

Then

$$
\mathscr{L}\left\{y_{2}\right\}=\frac{\lambda s^{2} I_{1}(s)-\frac{\lambda-E}{\ln 2} I_{0}(s)}{s^{2}-1},
$$

which is entire in $s$. Hence

$$
\lambda I_{1}(1)=\frac{\lambda-E}{\ln 2} I_{0}(1) .
$$

This determines $\frac{\lambda-E}{\lambda}$, and $\lambda$ itself is obtained from inverting $\mathscr{L}\left\{y_{2}\right\}$ and then evaluating $\Gamma y_{2}$. The integrals are not elementary, but are determinate. Among other things, we obtain in the process

$$
\int_{0}^{\infty} \frac{J_{0}(t) \sin x t}{1+t^{2}} d t=K_{0}(1) \sinh x \quad|x|<1 .
$$

We have used, fairly freely, the functions $f_{n}(x)$ for which $\Gamma f_{n}=x^{n}$. These are readily determined from

$$
\begin{gathered}
\Gamma f_{0}=1 \quad f_{0}=\frac{C}{\sqrt{1-x^{2}}} \\
C \int_{-1}^{1} \ln |x-t| \frac{d t}{\sqrt{1-t^{2}}}=1 \quad \text { giving } \quad C=-\frac{1}{\pi \ln 2} .
\end{gathered}
$$


Then, by (6),

$$
\begin{aligned}
\Gamma^{\prime} x f_{n} & =x \Gamma^{\prime} f_{n}-\int_{-1}^{1} f_{n} d t \\
& =x n x^{n-1}-\gamma_{n} \\
\Gamma x f_{n} & =\frac{n x^{n+1}}{n+1}-\gamma_{n} x+\delta_{n} . \\
& =\frac{n}{n+1} \Gamma f_{n+1}-\gamma_{n} \Gamma f_{1}+\delta_{n} \Gamma f_{n} .
\end{aligned}
$$

and the equation

$$
x f_{n}=\frac{n}{n+1} f_{n+1}-\gamma_{n} f_{1}+\delta_{n} f_{0}
$$

determines the remaining $f_{n}$ inductively.

3. The equation $\Gamma f=\int_{a}^{b} K_{0}(|x-t|) f(t) d t$.

We consider two cases for this equation. First, let $(a, b)=(0, \infty) .^{2)}$ Then, exactly as in (7),

$$
\Gamma^{\prime} y=\Gamma y^{\prime}
$$

for any $y(x)$, differentiable in $(0, \infty)$ and such that

$$
y(0)=0, \quad|y|=O\left(e^{\delta x}\right) \quad \delta<1 \quad \text { as } \quad x \rightarrow \infty .
$$

The kernel $K_{0}(|x|)$ satisfies a second order equation

$$
x K_{0}^{\prime \prime}(|x|)+K_{0}^{\prime}(|x|)-x K_{0}(|x|)=0,
$$

leading to

$$
\left(\Gamma^{\prime \prime}-\Gamma\right) x f=x\left(\Gamma^{\prime \prime}-\Gamma\right) f+\Gamma^{\prime} f
$$

replacing (6).

Starting with $\Gamma f=q(x)$, the computation of $\Gamma x f$ involves, instead of constants of integration, the functions $c_{1} e^{x}, c_{2} e^{-x}$. Accordingly, let us start with the equation

$$
\Gamma f=e^{-x}
$$

and look for $f(x)$ in the class $\{f(x)\}$ of functions which are integrable over $(0, a)$ for each $a$ bounded by $e^{\delta x}, \delta<1$, at infinity, and such that $x f(x) \rightarrow 0$

2)This case has been solved by means of the Wiener-Hopf procedure. 
as $x \rightarrow 0$. Again, the latter condition can be proven to hold, but the analysis is simpler if we assume it from the start. Computing $\Gamma x f$, we find

$$
\begin{gathered}
\left(\Gamma^{\prime \prime}-\Gamma\right) x f=-e^{-x} \\
\Gamma x f=\frac{x}{2} e^{-x}+A e^{-x}+B e^{x} .
\end{gathered}
$$

Now if

$$
f(x)=O\left(e^{\delta x}\right) \quad \text { for } \quad \delta<1
$$

then

$$
\begin{aligned}
\Gamma x f & =O\left(e^{\delta x}\right) \quad \text { also as } \quad x \rightarrow \infty, \quad \text { since } \\
K_{0}(|x|) & =O\left(e^{-x}\right) \quad \text { as } \quad x \rightarrow \infty .
\end{aligned}
$$

This gives $B=0$, and

$$
\Gamma x f=\frac{x}{2} e^{-x}+A e^{-x} .
$$

Choosing $y=x f$, the conditions of (17) are met, and thus

$$
\begin{aligned}
\Gamma^{\prime} x f=\Gamma(x f)^{\prime} & =\left(\frac{1}{2}-A\right) e^{-x}-\frac{x}{2} e^{-x} \\
& =\Gamma\left\{\left(\frac{1}{2}-A\right) f-x f+A f\right\},
\end{aligned}
$$

and from the uniqueness of the solution of the integral equation,

$$
(x f)^{\prime}=\left(\frac{1}{2}-x\right) f
$$

or

$$
f(x)=C \frac{e^{-x}}{\sqrt{x}}
$$

To solve $\Gamma g=1,0<x<\infty$, as another example we find

$$
\Gamma x g=x+B e^{-x} \text {. }
$$

Take $y=x g$, and as above

$$
\Gamma^{\prime} x g=1-B e^{-x}=\Gamma\{g-B f\}=\Gamma(x g)^{\prime}
$$

or

$$
x g^{\prime}=-B f .
$$

It becomes clear at this time that we are restricted to those elementary functions on the right hand side of $\Gamma f=q(x)$ for which the simple equation

$$
\left(D^{2}-1\right) \Gamma x f=x\left(D^{2}-1\right) \Gamma f+\Gamma^{\prime} f
$$


can be solved in closed form. We can handle $x^{n} e^{-k x}$, however, thereby obtaining solutions for a complete set of functions on the right.

So far, the examples considered have been solved by other methods. Let us now turn to a problem whose solution has not been obtained in closed form. Take

$$
\Gamma f=\int_{-1}^{1} K_{0}(|x-t|) f(t) d t
$$

(any finite interval being reducible to $(-1,1)$ by a simple change of variable). As a first step, we consider the special problems

$$
\begin{aligned}
& \Gamma f=\cosh x \\
& \Gamma g=\sinh x \\
& \Gamma h=1 \quad \text { all in } \quad-1<x<1 .
\end{aligned}
$$

It turns out to be simpler to consider the three equations simultaneously rather than separately. The identities already established still hold in the form

$$
\begin{gathered}
\left(\Gamma^{\prime \prime}-\Gamma\right) x f=x\left(\Gamma^{\prime \prime}-\Gamma\right) f+\Gamma^{\prime} f \\
\Gamma^{\prime} y=\Gamma y^{\prime} \quad \text { if } \quad y(-1)=y(1)=0 \quad(\text { see }(7)) .
\end{gathered}
$$

We know immediately that $f(x), h(x)$ are even in $x$, while $g(x)$ is odd. Computing $\Gamma x f, \cdots, \Gamma x^{2} h$,

$$
\begin{aligned}
\Gamma f & =\cosh x, \\
\Gamma x f & =\frac{x}{2} \cosh x+A \sinh x, \\
\Gamma x^{2} f & =\frac{3}{8} x^{2} \cosh x+\left(\frac{A}{2}-\frac{1}{8}\right) x \sinh x+C \cosh x, \\
\Gamma g & =\sin x, \\
\Gamma x g & =\frac{x}{2} \sinh x+B \cosh x, \\
\Gamma x^{2} g & =\frac{3}{8} x^{2} \sinh x+\left(\frac{B}{2}-\frac{1}{8}\right) x \cosh x+D \sinh x, \\
\Gamma h & =1 \\
\Gamma x h & =x+\alpha \sinh x, \\
\Gamma x^{2} h & =1+x^{2}+\frac{\alpha}{2} x \sinh x+\beta \cosh x .
\end{aligned}
$$


Next, let $y_{1}, \cdots, y_{6}$ be defined by

$$
\begin{aligned}
& y_{1}=\left(1-x^{2}\right) f, \quad y_{2}=\left(1-x^{2}\right) g, \quad y_{3}=\left(1-x^{2}\right) h \\
& y_{4}=\int_{-1}^{x} t f d t, \quad y_{5}=\int_{-1}^{x} g d t, \quad y_{6}=\int_{-1}^{x} t h d t .
\end{aligned}
$$

From (20) we have, for example,

$$
\begin{aligned}
\Gamma^{\prime} y_{5} & =\Gamma y_{5}^{\prime}=\Gamma g=\sinh x, \\
\Gamma y_{5} & =\cosh x+\mu=\Gamma(f+\mu h) .
\end{aligned}
$$

Thus

$$
\begin{aligned}
& y_{5}=f+\mu h, \\
& y_{5}^{\prime}=g,
\end{aligned}
$$

and

$$
f^{\prime}+\mu h^{\prime}=g^{\prime}
$$

Repeating this procedure for $y_{1}, \cdots, y_{6}$, we obtain the six equations

$$
\begin{gathered}
\left(1-x^{2}\right) f^{\prime}=\left(\rho-x^{2}\right) g+x f\left(B-A+\frac{1}{2}\right), \\
\left(1-x^{2}\right) g^{\prime}=\left(\eta-x^{2}\right) f+x g\left(A-B+\frac{1}{2}\right), \\
\left(1-x^{2}\right) h^{\prime}=\left(\frac{3 \alpha}{2}-\beta+A \alpha\right) g-\alpha x f, \\
x g^{\prime}+g+\left(A-B-\frac{1}{2}\right) f^{\prime}+\lambda h^{\prime}=x f, \\
\quad f^{\prime}+\mu h^{\prime}=g, \\
\left(x^{2}+2 \nu-1\right) h^{\prime}=\alpha\left(x g^{\prime}+g\right)+(\beta-\alpha B-2 \alpha) f^{\prime},
\end{gathered}
$$

where

$$
\begin{aligned}
& \rho=A^{2}+A-A B+D-C+\frac{9}{8}, \\
& \eta=B^{2}+B-A B+C-D+\frac{9}{8}
\end{aligned}
$$

we also set $A-B-\frac{1}{2}=\xi$.

Any three independent equations from this set determine $f, g$, and $h$. However, each equation involves parameters which depend on the solutions of the equations, and at present, all we can obtain are the consistency relations between the equations, and hence certain equations involving the parameters. Of these, only one appears to be of value, and we derive it as follows. Eliminate $h^{\prime}, f^{\prime}, g^{\prime}$ from the first, second, fourth, and fifth of these equations, and we obtain

$$
C_{1} x f=\left(C_{2}+C_{3} x^{2}\right) g
$$


and as is readily verified from the relations for $\Gamma x f, \Gamma g, \Gamma x^{2} g$, we must have

$$
C_{1}=C_{2}=C_{3}=0 \text {. }
$$

In expanded form, these are

$$
\begin{aligned}
\lambda(1-\rho)+\mu(1+\rho \xi) & =0 \\
-\lambda \xi+\mu\left\{1-\eta+\xi^{2}\right\} & =0 .
\end{aligned}
$$

Thus, either $\lambda=\mu=0$, or the determinant of this system,

$$
(1-\rho)(1-\eta)+\xi(1+\xi),
$$

must vanish. The possibility $\lambda=\mu=0$ leads to $x f^{\prime \prime}+(1+\xi) f^{\prime}-x f=0$, and $y_{5}=f,=0$ at \pm 1 , implying $f(x)=0$. We have then

$$
(1-\rho)(1-\eta)+\xi(1+\xi)=0 \text {. }
$$

This condition, together with

$$
\begin{gathered}
\left(1-x^{2}\right) f^{\prime}+\xi x f=\left(\rho-x^{2}\right) g \\
\left(1-x^{2}\right) g^{\prime}-(1+\xi) x g=\left(\eta-x^{2}\right) f
\end{gathered}
$$

constitutes an eigenvalue problem for the functions $f, g$, with eigenvalues $\rho, \eta$, the eigenconditions being that $f(x)$ is even, $g(x)$ is odd, and both behave like $1 / \sqrt{1-x^{2}}$ in a neighborhood of $x= \pm 1$. To verify this latter statement, we observe that $y_{4}, y_{5}, y_{6}$ give the useful information that certain combinations of $f, g, h$ vanish at $x= \pm 1$. In particular, then, $f(x)$ and $g(x)$ have the same behavior there, and the indicial equation of (22) at $x= \pm 1$ becomes

$$
2 \sigma(1+2 \sigma)-(1-\rho)(1-\eta)-\xi(1+\xi)=0
$$

so that, with (21), we have $\sigma=0$ or $\sigma=-\frac{1}{2}$. As is well known, the correct value is $\sigma=-\frac{1}{2}$.

It may be convenient to replace $f(x)$ by $F(x) / \sqrt{1-x^{2}}, g(x)$ by $G(x) / \sqrt{1-x^{2}}$, and we have

$$
\begin{aligned}
& \left(1-x^{2}\right) F^{\prime}+(1+\xi) x F=\left(\rho-x^{2}\right) G, \\
& \left(1-x^{2}\right) G^{\prime}-\xi x G=\left(\eta-x^{2}\right) F
\end{aligned}
$$

with $F$ even, $G$ odd, and both analytic near $x= \pm 1$.

Unfortunately, the system (23), or the single equation obtained on elimination of one of $F, G$, is not solvable in terms of elementary, or even well known, functions. The system has two parameters, and undoubtedly a sequence of pairs of eigenvalues $\left(\rho_{n}, \eta_{n}\right)$ with solution pairs $F_{n}, G_{n}$. In this case, we require the 
values $\rho_{1}, \eta_{1}$, and $F_{1}, G_{1}$ so that $F_{1}(x)$ has no zeros in $-1 \leqq x \leqq 1$. Partial results have been obtained, but a complete description of the solution is still to be found.

At any rate, the original integral equation has been replaced by a system of ordinary differential equations, and for this system, many more techniques are available for determining the solutions.

5. Conclusion. The kernels in each of the integral equations considered here have satisfied linear differential equations with linear coefficients. In a formal way, we can establish our identities for any such kernel. Thus if

$$
\begin{aligned}
& \Gamma f=r(x), \\
& \Gamma f \equiv \int_{a}^{b} K(x-t) f(t) d t,
\end{aligned}
$$

where $K(u)$ satisfies

$$
u L K(u)+M K(u)=0,
$$

with $L, M$ being ordinary differential operators with constant coefficients, we have

$$
\Gamma x f=-\int_{a}^{b}(x-t) K(x-t) f d t+x \Gamma f
$$

and

$$
L(\Gamma x f)=M \Gamma f+x L(\Gamma f) .
$$

If we have uniqueness, then, the solution of $\Gamma f=r(x)$, for elementary $r(x)$, can be expressed in terms of the solutions of $\Gamma g=s_{i}(x)$, where $L\left(s_{i}(x)\right)=0$.

Unfortunately, there seems to be no simple method of handling cases for which the differential equation satisfied by $K(u)$ has other than linear coefficients.

So far, we have considered only cases for which the solutions of the integral equations are functions of a single variable and accordingly lead to ordinary differential equations. Some higher dimensional cases can be handled by precisely the same technique and will be discussed in a later paper.

Prepared under contract Nonr 225(11) with Stanford University. 